\title{
An Estimation of a Hybrid Log-Poisson Regression Using a Quadratic Optimization Program for Optimal Loss Reserving in Insurance
}

\author{
Apollinaire Woundjiagué $\left(\mathbb{D}\right.$, 1,2 Martin Le Doux Mbele Bidima $(\mathbb{D}){ }^{3}$ \\ and Ronald Waweru Mwangi ${ }^{4}$ \\ ${ }^{1}$ Institute of Basic Sciences Technology and Innovation, \\ Pan African University-Jomo Kenyatta University of Agriculture and Technology, P.O. Box 62000-00200, Nairobi, Kenya \\ ${ }^{2}$ National Advanced School of Engineering, University of Maroua, P.O. Box 46 Maroua, Cameroon \\ ${ }^{3}$ Faculty of Science, University of Yaounde I, P.O. Box 812, Yaounde, Cameroon \\ ${ }^{4}$ School of Computing and Information Technology, Jomo Kenyatta University of Agriculture and Technology, \\ P.O. Box 62000-00200, Nairobi, Kenya
}

Correspondence should be addressed to Apollinaire Woundjiagué; appoappolinaire@yahoo.fr

Received 15 December 2018; Revised 7 March 2019; Accepted 20 May 2019; Published 16 June 2019

Academic Editor: Patricia Melin

Copyright (C) 2019 Apollinaire Woundjiagué et al. This is an open access article distributed under the Creative Commons Attribution License, which permits unrestricted use, distribution, and reproduction in any medium, provided the original work is properly cited.

In this article, we are interested in developing an alternative estimation method of the parameters of the hybrid log-Poisson regression model. In our previous paper, we have proposed a hybrid log-Poisson regression model where we have derived the analytical expression of the fuzzy parameters. We found that the hybrid model provide better results than the classical log-Poisson regression model according to the mean square error prediction and the goodness of fit index. However, nowhere we have taken into account the optimal value of $h(\alpha$-cut) which is of greatest importance in fuzzy regressions literature. In this paper, we provide an alternative estimation method of our hybrid model using a quadratic optimization program and the optimized $h$-value ( $\alpha$-cut). The expected value of fuzzy number is used as a defuzzification procedure to move from fuzzy values to crisp values. We perform the hybrid model with the alternative estimation we are suggesting on two different numerical data to predict incremental payments in loss reserving. From the mean square error prediction, we prove that the alternative estimation of the new hybrid model with an optimized $h$-value predicts incremental payments better than the classical log-Poisson regression model as well as the same hybrid model with analytical estimation of parameters. Hence we have optimized the outstanding loss reserves.

\section{Introduction}

"An important role of a non-life actuary is the calculation of provisions, mainly IBNR (incurred but not reported) reserve. Then, finding the fair value of loss reserve is a relevant topic for non-life actuaries. Indeed, insurance companies must simultaneously have enough reserves to meet their commitment to policyholders and have enough funds for their investments. Therefore several methods have been proposed in actuarial science literature to capture this fair value" [1].

"In one hand, we distinguish deterministic methods [24]. They provide crisp predictions for reserves. In [sic] the other hand, [5-8] present stochastic methods. Those methods don't give only a crisp value of the reserves but provide also their variability. For more details on existing loss reserving methods, one can consult [9-19]" [1].

But in [20], there are some experiences where stochastic methods can give unrealistic estimates. For example, when the claims are related to body injures, the future losses for the company will depend on the growth of the wage index that helps to determine the amount of indemnity and depends also on changes in court practices and public awareness of liability matters. Then the information is vague. Therefore the use of Fuzzy Set Theory becomes very attractive when the information is vague as in this case. Hence one should 
think about models that can handle both fuzziness and randomness, namely, hybrid models.

In [1], we have proved that we can improve the classical log-Poisson regression model through a hybrid one where the parameters are derived and have an analytical form. Although the new model provides better results than the classical logPoisson regression model (according to mean square error prediction (MSEP) and goodness of fit index), we still have a large value of the MSEP. This could be due to the choice of $h$-value which is important in fuzzy regression framework. The purpose of this paper is to provide an alternative estimation which is taking into account the optimized $h$-value. We will prove in this paper that the optimized $h$-value is of the greatest importance because the value of the MSEP will be very low compared to the MSEP we are getting from the analytical estimation of the hybrid model.

In this paper, we investigate the possibilistic approach to estimate the hybrid model, i.e., to estimate the asymmetric triangular fuzzy coefficients (ATFC) of the model through a quadratic optimization program and by taking into account an optimized value of $h$ ( $\alpha$-cut) in loss reserving framework. We have investigated the fuzzy least-squares approach in our previous paper [1]. To move from FN to crisp values, we shall use the expected value of FN.

Our objective therefore is to come with a new estimation method of fuzzy parameters in the hybrid log-Poisson model where the optimized $h$-value will be taken into account. From two different data, we prove that our hybrid model the new estimation method provides best predictions of reserves compared to the classical log-Poisson model according to the MSEP criterion.

The structure of the paper is as follows: We present in the first section the preliminaries on fuzzy sets and their properties. In the second section, we shall review some models and results on FRM. In the third section, the framework of estimation of loss reserve with log-Poisson regression will be introduced. Our contribution starts from Section 5 where we propose a new estimation method of the hybrid log-Poisson regression model [1] for loss reserving through a quadratic optimization program by taking into account the optimized $h$-value and we prove its relevance from two datasets. Then we conclude the article.

\section{Preliminaries on Fuzzy Sets and Their Properties}

In this section, we review some concepts related to our research. That is the concept of fuzzy set, membership function, FN, FRM.

\subsection{Review of Some Definitions and Properties of Fuzzy Sets}

Definition 1 (from [21]). Let $\Omega$ be a nonempty set and $\omega \in \Omega$. In classical set theory, a subset $A$ of $\Omega$ can be defined by its characteristic function $\chi_{A}$ as a mapping from the elements of $\Omega$ to the elements of the set $\{0,1\}$,

$$
\chi_{A}: \Omega \longrightarrow\{0,1\}
$$

This mapping may be represented as a set of ordered pairs, with exactly one ordered pair present for each element of $\Omega$. The first element of the ordered pair is an element of the set $\Omega$, and the second element is an element of the set $\{0,1\}$. The value zero is used to represent nonmembership, and the value one is used to represent membership. The truth or falsity of the statement " $\omega$ is in $A$ " is determined by the ordered pair $\left(\omega, \chi_{A}(\omega)\right)$. The statement is true if the second element of the ordered pair is 1 , and the statement is false if it is 0 .

Similarly, a fuzzy subset (also called fuzzy set) $\widetilde{A}$ of a set $\Omega$ can be defined as a set of ordered pairs, each with the first element from $\Omega$ and the second element from the interval $[0,1]$, with exactly one ordered pair present for each element of $\Omega$. This defines a mapping called membership function.

Definition 2 (from [21]). The membership function of a fuzzy set $\widetilde{A}$, denoted by $\mu_{\widetilde{A}}$, is defined by

$$
\mu_{\widetilde{A}}: \Omega \longrightarrow[0,1]
$$

where $\mu_{\widetilde{A}}$ is typically interpreted as the membership degree of element $\omega$ in the fuzzy set $\widetilde{A}$.

The degree to which the statement " $\omega$ is in $\widetilde{A}$ " is true is determined by finding the ordered pair $\left(\omega, \mu_{\widetilde{A}}(\omega)\right)$. The degree of truth of the statement is the second element of the ordered pair. A fuzzy set $\widetilde{A}$ on $\Omega$ can also be defined as a set of tuples

$$
\widetilde{A}=\left\{\left(\omega, \mu_{\widetilde{A}}(\omega)\right) \mid \omega \in \Omega\right\}
$$

and could be represented by a graphic.

Definition 3 (from [22]). Let $\Omega$ be the set of objects and $\widetilde{A} \subset$ $\Omega$. The $\alpha$-cut $\widetilde{A}_{\alpha}$ of $\widetilde{A}$ is the set defined by

$$
\widetilde{A}_{\alpha}=\left\{\omega \in \Omega, \mu_{\widetilde{A}}(\omega) \geqslant \alpha\right\} .
$$

Definition 4 (from [23]).

(1) A FN $\widetilde{A}$ is a fuzzy set of a universe $\Omega$ (the real line $\mathbb{R}$ ) such that

(a) all its $\alpha$-cut are convex which is equivalent to the fact that $\widetilde{A}$ is convex, that is, $\forall \omega_{1}, \omega_{2} \in$ $\mathbb{R}$ and $\lambda \in[0,1], \quad \mu_{\widetilde{A}}\left(\lambda \omega_{1}+(1-\lambda) \omega_{2}\right) \geqslant$ $\min \left(\mu_{\widetilde{A}}\left(\omega_{1}\right), \mu_{\widetilde{A}}\left(\omega_{2}\right)\right) ;$

(b) $\widetilde{A}$ is normalized, that is, $\exists \omega_{0} \in \Omega$ such that $\mu_{\widetilde{A}}\left(\omega_{0}\right)=1$.

(c) $\mu_{\widetilde{A}}$ is continued membership function of bounded support, where $\Omega=\mathbb{R}$ and $[0,1]$ are equipped with the natural topology.

(2) A triangular fuzzy number (TFN) $\tilde{\gamma}$ is a FN denoted by $\tilde{\gamma}=\left(\beta^{L}, \alpha^{c}, \beta^{R}\right) ; \beta^{L}, \alpha^{c}, \beta^{R} \in \mathbb{R}$, such that $\mu_{\widetilde{A}}\left(\beta^{L}\right)=$ $\mu_{\widetilde{A}}\left(\beta^{R}\right)=0$ and $\mu_{\widetilde{A}}\left(\alpha^{c}\right)=1$ with $\alpha^{c}$ the centre of $\widetilde{\gamma}, \beta^{L}$ its left spread and $\beta^{R}$ its right spread [24]. 


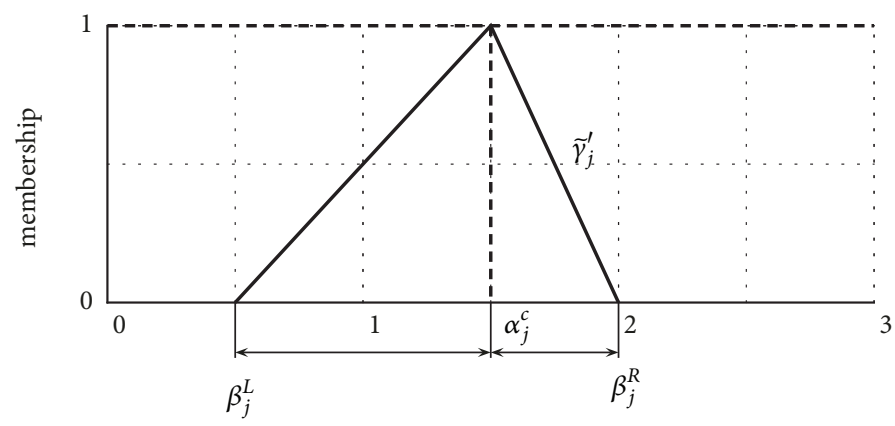

Figure 1: An example of Asymmetric Triangular Fuzzy Number $\left(\widetilde{\gamma}_{j}=\left(\beta_{j}^{L}, \alpha_{j}^{c}, \beta_{j}^{R}\right)\right)$.

A TFN $\tilde{\gamma}$ could be defined with its membership degree function $\mu_{\tilde{\gamma}}$ or, with its $h$-level $\left(\alpha-\right.$ cut $(h \in[0,1]) \gamma_{h}$ (see [23]), i.e.,

$$
\mu_{\tilde{\gamma}}(x)= \begin{cases}1-\frac{\alpha^{c}-x}{\beta^{L}} & \text { if } \alpha-\beta^{L}<x \leqslant \alpha \\ 1-\frac{x-\alpha^{c}}{\beta^{R}} & \text { if } \alpha<x \leqslant \alpha+\beta^{R} \\ 0 & \text { if otherwise }\end{cases}
$$

or

$$
\widetilde{\gamma}_{h}=\left[\gamma_{L_{h}}, \gamma_{R_{h}}\right]=\left[\alpha^{c}-\beta^{L}(1-h), \alpha^{c}+\beta^{R}(1-h)\right]
$$

(i) If $\alpha^{c}-\beta^{L}=\beta^{R}-\alpha^{c}$, then $\tilde{\gamma}$ define a STFN

(ii) Otherwise $\beta^{L} \neq \beta^{R}$; then $\tilde{\gamma}$ define an ATFN (see Figure 1).

(1) Notes and Comments. It is well know that if $\widetilde{A}$ is a FN, then $\widetilde{A}_{h}$, the $h$ level ( $\alpha$-cut) of $\widetilde{A}$, is a compact set of $\mathbb{R}$, for all $h \in[0,1]$.

Let us present some properties on TFN.

Property 5 (from [25]). Let $f(\widetilde{\boldsymbol{\Gamma}})=f\left(\tilde{\gamma}_{1}, \tilde{\gamma}_{2}, \ldots, \tilde{\gamma}_{k}\right)$ be a $k$-vector of TFN such that $\tilde{\gamma}_{i}=\left(\beta_{i}^{L}, \alpha_{i}^{c}, \beta_{i}^{R}\right) \in \mathbb{R}^{3}, i=1, \ldots, k$, are TFN.

(1) If $f(\widetilde{\boldsymbol{\Gamma}})$ is obtained from a linear combination of the TFN $\tilde{\gamma}_{i}, i=1, \ldots, k$, then $f(\widetilde{\Gamma})=$ $\left(f^{L}(\Gamma), f^{c}(\Gamma), f^{R}(\Gamma)\right)$ is also a TFN, where

$$
\begin{aligned}
f^{L}(\Gamma) & =\sum_{\substack{i=1 \\
r_{i} \geqslant 0}}^{k} \beta_{i}^{L}\left|r_{i}\right|+\sum_{\substack{i=1 \\
r_{i}<0}}^{k} \beta_{i}^{R}\left|r_{i}\right| \\
f^{c}(\Gamma) & =\sum_{i=1}^{k} \alpha_{i}^{c} r_{i} \\
f^{R}(\Gamma) & =\sum_{\substack{i=1 \\
r_{i} \geqslant 0}}^{k} \beta_{i}^{R}\left|r_{i}\right|+\sum_{\substack{i=1 \\
r_{i}<0}}^{N} \beta_{i}^{L}\left|r_{i}\right|
\end{aligned}
$$

From the extension principle [26-28], we can obtain the $h$ - level of $f(\widetilde{\Gamma})$, i.e.,

$$
[f(\Gamma)]_{h}=f\left(\gamma_{1_{h}}, \gamma_{2_{h}}, \ldots, \gamma_{k_{h}}\right)
$$

and

$$
\begin{aligned}
f(\widetilde{\boldsymbol{\Gamma}}) & =\sum_{i=1}^{k} r_{i} \widetilde{\gamma}_{i} \\
& =\left(f^{L}(\Gamma), f^{\mathcal{c}}(\Gamma), f^{R}(\Gamma)\right), \quad \text { with } r_{i} \in \mathbb{R}
\end{aligned}
$$

(2) If $f(\widetilde{\boldsymbol{\Gamma}})$ is evaluated by nonlinear functions with TFN, i.e., $f(\cdot)$ is increasing with respect to the first $n$ variables, where $n \leqslant k$, and decreasing with respect to $k-n$ variables, the result will not be a TFN. But [23] has shown that $f(\widetilde{\boldsymbol{\Gamma}})$ can be approximate with a $\operatorname{TFN} f^{\prime}(\widetilde{\Gamma})=\left(f^{L^{\prime}}(\Gamma), f^{c^{\prime}}(\Gamma), f^{R^{\prime}}(\Gamma)\right)$, i.e.,

$$
\begin{aligned}
f^{L^{\prime}}(\Gamma) & =\sum_{i=1}^{n} \frac{\partial f^{c}(\Gamma)}{\partial \alpha_{i}^{c}} \beta_{i}^{L}-\sum_{i=n+1}^{k} \frac{\partial f^{c}(\Gamma)}{\partial \alpha_{i}^{c}} \beta_{i}^{R} \\
f^{c^{\prime}}(\Gamma) & =f\left(\alpha_{1}^{c}, \alpha_{2}^{c}, \ldots, \alpha_{k}^{c}\right)=f^{c}(\Gamma) \\
f^{R^{\prime}}(\Gamma) & =\sum_{i=1}^{n} \frac{\partial f^{c}(\Gamma)}{\partial \alpha_{i}^{c}} \beta_{i}^{R}-\sum_{i=n+1}^{k} \frac{\partial f^{c}(\Gamma)}{\partial \alpha_{i}^{c}} \beta_{i}^{L}
\end{aligned}
$$

In the next section, we review some results on fuzzy linear regression in the literature.

\section{Review of Some Models and Results on Fuzzy Regression}

In this section we review the FRM proposed by [29] and the one proposed by [30]; then we present their properties. These models will help us to develop the new hybrid model for loss reserving.

3.1. Ishibuchi's FRM with Asymmetric Triangular Fuzzy Coefficients. Let us define the fuzzy linear regression (FLR) model proposed by [29].

Let $\left(\mathbf{Y}_{N \times 1}, \mathbf{X}_{N \times(m+1)}\right)$ be the given crisp data and let

$$
\widetilde{\mathbf{Y}}_{N \times 1}=f\left(\mathbf{X}_{N \times(m+1)}\right)=\mathbf{X}_{N \times(m+1)} \otimes \widetilde{\mathbf{\Gamma}}_{(m+1) \times 1}
$$




$$
\begin{aligned}
\left(\begin{array}{c}
\tilde{Y}_{1} \\
\vdots \\
\tilde{Y}_{N}
\end{array}\right) & =\left(\begin{array}{c}
\mathbf{x}_{1} \\
\vdots \\
\mathbf{x}_{N}
\end{array}\right) \otimes\left(\begin{array}{c}
\tilde{\gamma}_{0} \\
\vdots \\
\tilde{\gamma}_{m}
\end{array}\right) \\
& =\left(\begin{array}{c}
\mathbf{x}_{1} \\
\vdots \\
\mathbf{x}_{N}
\end{array}\right) \otimes\left(\begin{array}{c}
\left(\alpha_{0}^{c}, \beta_{0}\right) \\
\vdots \\
\left(\alpha_{m}^{c}, \beta_{m}\right)
\end{array}\right) \\
& =\left(\begin{array}{c}
\left(\sum_{j=0}^{m} \alpha_{j}^{c} x_{1 j}, \sum_{j=0}^{m} \beta_{j} x_{1 j}\right) \\
\vdots \\
\left(\sum_{j=0}^{m} \alpha_{j}^{c} x_{N j}, \sum_{j=0}^{m} \beta_{j} x_{N j}\right)
\end{array}\right)
\end{aligned}
$$

be a FLR model with STFC, where $\widetilde{\mathbf{Y}}$ is a fuzzy output from $f\left(\mathbf{X}_{N \times(m+1)}\right), \mathbf{X}_{N \times(m+1)}$ is the matrix of the given crisp dataset, and $\widetilde{\boldsymbol{\Gamma}}_{(m+1) \times 1}=\left[\left(\alpha_{0}^{c}, \beta_{0}\right),\left(\alpha_{1}^{c}, \beta_{1}\right), \ldots,\left(\alpha_{m}^{c}, \beta_{m}\right)\right]^{T}$ is the fuzzy parameter of the model.

In model (16), $\widetilde{\gamma}_{j}=\left(\alpha_{j}^{c}, \beta_{j}\right) \in \mathbb{R}^{2}, j=0, \ldots, m$ are fuzzy coefficients such that $\alpha_{j}^{c}$ are the centres of $\widetilde{\gamma}_{j}$, and $\beta_{j}$ are its spreads. The disturbance term is not introduced as a random addend in the linear relation, but incorporated into the coefficients $\widetilde{\gamma}_{j}, j=0, \ldots, m$. STFN.

When the coefficients are STFN, the output $\widetilde{\mathbf{Y}}$ is also a
Let us denote

$$
\begin{aligned}
f\left(X_{i}\right) & =\left(f^{\alpha^{c}}\left(X_{i}\right), f^{\beta}\left(X_{i}\right)\right), \quad i=1, \ldots, N \\
f\left(\mathbf{X}_{N \times(m+1)}\right) & =\left[f\left(X_{1}\right), \ldots, f\left(X_{N}\right)\right]^{T}
\end{aligned}
$$

where

$$
\begin{aligned}
f^{\alpha^{c}}\left(X_{i}\right) & =\alpha_{0}^{c}+\sum_{j=1}^{m} \alpha_{j}^{c} \cdot x_{i j} \\
f^{\beta}\left(X_{i}\right) & =\beta_{0}+\sum_{j=1}^{m} \beta_{j} \cdot\left|x_{i j}\right|, \quad i=1, \ldots, N
\end{aligned}
$$

and $X_{i}=\left(x_{i 1}, x_{i 2}, \ldots, x_{i m}\right)$.

Then $f\left(X_{i}\right)$ is a STFN. Its $h$-level, i.e., $\alpha$-cut with $\alpha=h$ is calculated as follows for $h \in[0,1]$ :

$$
\begin{aligned}
& {\left[f\left(X_{i}\right)\right]_{h}=\left[f^{\alpha^{c}}\left(X_{i}\right)-(1-h) \cdot f^{\beta}\left(X_{i}\right), f^{\alpha^{c}}\left(X_{i}\right)\right.} \\
& \left.\quad+(1-h) \cdot f^{\beta}\left(X_{i}\right)\right], \quad i=1, \ldots, N
\end{aligned}
$$

From (24), $Y_{i} \in\left[f\left(X_{i}\right)\right]_{h}, i=1, \ldots, N \Longrightarrow$

$$
\begin{aligned}
& f^{\alpha^{c}}\left(X_{i}\right)-(1-h) \cdot f^{\beta}\left(X_{i}\right) \leqslant Y_{i} \\
& f^{\alpha^{c}}\left(X_{i}\right)+(1-h) \cdot f^{\beta}\left(X_{i}\right) \geqslant Y_{i}, \quad i=1,2, \ldots, N
\end{aligned}
$$

To determine the parameters $\widetilde{\gamma}_{j}=\left(\alpha_{j}^{c}, \beta_{j}\right)$ of the FRM (16), [29] proposes to solve a linear programming problem with an objective function of minimizing the total spread of the fuzzy coefficient, i.e.,

$$
\begin{aligned}
\min : & z=\sum_{i=1}^{N} f^{\beta}\left(X_{i}\right)=\sum_{i=1}^{N}\left\{\beta_{0}+\beta_{1} \cdot\left|x_{i 1}\right|+\ldots+\beta_{m} \cdot\left|x_{i m}\right|\right\}, \\
\text { subject to } & f^{\alpha^{c}}\left(X_{i}\right)-(1-h) \cdot f^{\beta}\left(X_{i}\right) \leqslant Y_{i} \\
& f^{\alpha^{c}}\left(X_{i}\right)+(1-h) \cdot f^{\beta}\left(X_{i}\right) \geqslant Y_{i}, \\
& \beta_{j} \geqslant 0, \quad i=1, \ldots, N ; j=0,1, \ldots, m
\end{aligned}
$$

Reference [30] has shown that the fuzzy regression method developed by [29], when applied to different data sets, can provide the same FRM by solving the linear programming problem in (26) for $h=0.5$. Then the authors proposed the FRM with ATFC in order to remedy this limitation.

Let us assume that

$$
\widetilde{\gamma}_{j}=\left(\beta_{j}^{L}, \alpha_{j}^{c}, \beta_{j}^{R}\right), \quad j=0,1, \ldots, m
$$

is ATFC in the fuzzy model (16), where $\beta_{j}^{L}$ is its left spread, $\alpha_{j}^{c}$ its centre, and $\beta_{j}^{R}$ its right spread (see Figure 1).

When $\forall j=0,1, \ldots, m, \widetilde{\gamma}_{j}$ are ATFC in model (16); then $f\left(\mathbf{X}_{N \times(m+1)}\right)$ is also calculated as an ATFC [30]. Hence, we denote

$$
\begin{aligned}
f\left(\mathbf{X}_{N \times(m+1)}\right)=\left(\begin{array}{c}
f\left(X_{1}\right) \\
\vdots \\
f\left(X_{N}\right)
\end{array}\right) \\
=\left(\begin{array}{c}
\left(f^{\beta^{L}}\left(X_{1}\right), f^{\alpha^{c}}\left(X_{1}\right), f^{\beta^{R}}\left(X_{1}\right)\right) \\
\vdots \\
\left(f^{\beta^{L}}\left(X_{N}\right), f^{\alpha^{c}}\left(X_{N}\right), f^{\beta^{R}}\left(X_{N}\right)\right)
\end{array}\right)
\end{aligned}
$$

From [31], $f^{\beta^{L}}\left(X_{i}\right), f^{\alpha^{c}}\left(X_{i}\right)$ and $f^{\beta^{R}}\left(X_{i}\right), i=1, \ldots, N$, are compute as follows: 


$$
\begin{aligned}
& f^{\beta^{L}}\left(X_{i}\right)=\beta_{0}^{L}+\sum_{\substack{j=1 \\
x_{i j} \geqslant 0}}^{m} \beta_{j}^{L} \cdot x_{i j}+\sum_{\substack{j=1 \\
x_{i j}<0}}^{m} \beta_{j}^{R} \cdot x_{i j} \\
& f^{\alpha^{c}}\left(X_{i}\right)=\alpha_{0}^{c}+\sum_{j=1}^{m} \alpha_{j}^{c} \cdot x_{i j} \\
& f^{\beta^{R}}\left(X_{i}\right)=\beta_{0}^{R}+\sum_{\substack{j=1 \\
x_{i j} \geqslant 0}}^{m} \beta_{j}^{R} \cdot x_{i j}+\sum_{\substack{j=1 \\
x_{i j}<0}}^{m} \beta_{j}^{L} \cdot x_{i j}
\end{aligned}
$$

and the $h$-level of $f\left(X_{i}\right)$ is as follows:

$$
\begin{gathered}
{\left[f\left(X_{i}\right)\right]_{h}=\left[h \cdot f^{\alpha^{c}}\left(X_{i}\right)+(1-h) \cdot f^{\beta^{L}}\left(X_{i}\right), h\right.} \\
\left.\cdot f^{\alpha^{c}}\left(X_{i}\right)+(1-h) \cdot f^{\beta^{R}}\left(X_{i}\right)\right] \quad i=1, \ldots, N
\end{gathered}
$$

The steps to determine the fuzzy coefficients in (27) are as follows [30]:

(i) determine $f^{\alpha^{c}}\left(X_{i}\right) i=1, \ldots, N$ by OLS,

(ii) determine $f^{\beta^{L}}\left(X_{i}\right), f^{\beta^{R}}\left(X_{i}\right)$ of $f\left(X_{i}\right)$ for $i=1, \ldots, N$ by solving the linear programming problem:

$$
\begin{aligned}
& \min : \quad z=\sum_{i=1}^{N}\left\{f^{\beta^{R}}\left(X_{i}\right)-f^{\beta^{L}}\left(X_{i}\right)\right\}, \\
& \text { subject to } h \cdot f^{\alpha^{c}}\left(X_{i}\right)+(1-h) \cdot f^{\beta^{L}}\left(X_{i}\right) \leqslant Y_{i} \\
& h \cdot f^{\alpha^{c}}\left(X_{i}\right)+(1-h) \cdot f^{\beta^{R}}\left(X_{i}\right) \geqslant Y_{i}, \quad i=1,2, \ldots, N \\
& \beta_{j}^{L} \leq \alpha_{j}^{c} \leq \beta_{j}^{R}, \quad j=0,1, \ldots, m
\end{aligned}
$$

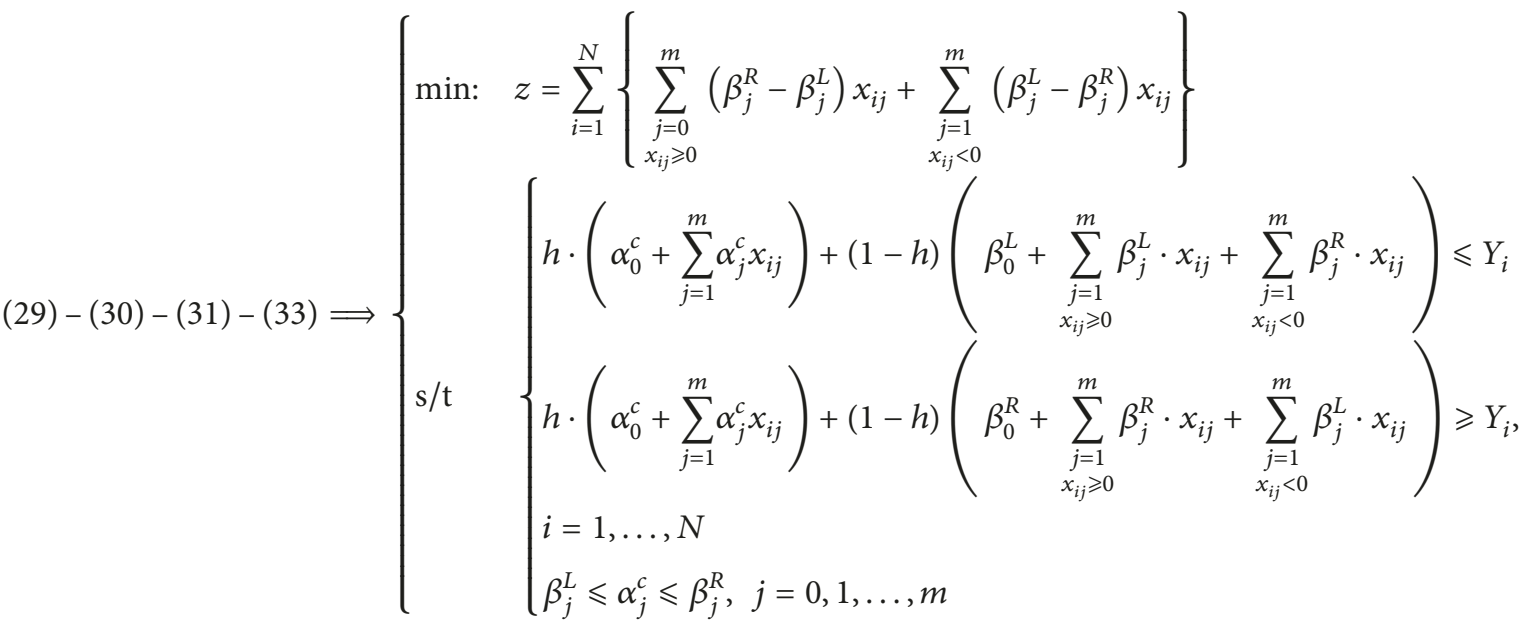

3.2. Optimizing the $h$ Value for FLR with ATFC. Let us present in this subsection the optimising $h$ value for ATFC developed by [32] that we shall use later to compute an optimized outstanding loss reserves.

3.2.1. Preliminaries and Some Definitions. Let us consider the model (16), but by considering

$$
\begin{aligned}
& \widetilde{\boldsymbol{\Gamma}}_{(m+1) \times 1}=\left[\widetilde{\gamma}_{0}, \widetilde{\gamma}_{1}, \ldots, \widetilde{\gamma}_{m}\right]^{T} \\
& \quad=\left[\left(\beta_{0}^{L}, \alpha_{0}^{c}, \beta_{0}^{R}\right),\left(\beta_{1}^{L}, \alpha_{1}^{c}, \beta_{1}^{R}\right), \ldots,\left(\beta_{m}^{L}, \alpha_{m}^{c}, \beta_{m}^{R}\right)\right]^{T} .
\end{aligned}
$$

That is a FLR with ATFC.

The system fuzziness in this case (model (16) with (35)) is defined by

$$
\begin{aligned}
& \nabla=\sum_{i=1}^{N} \nabla \tilde{Y}_{i} \\
& =\frac{1}{2} \sum_{i=1}^{N}\left(f^{\beta^{R}}\left(\mathbf{x}_{i}\right)-f^{\beta^{L}}\left(\mathbf{x}_{i}\right)\right) \\
& =\frac{1}{2} \sum_{i=1}^{N}\left(\sum_{\substack{j=1 \\
x_{i j} \geqslant 0}}^{m}\left(\beta_{j}^{L}+\beta_{j}^{R}\right) x_{i j}-\sum_{\substack{j=1 \\
x_{i j}<0}}^{m}\left(\beta_{j}^{L}+\beta_{j}^{R}\right) x_{i j}\right. \\
& \left.+\left(\beta_{0}^{R}-\beta_{0}^{L}\right)\right)
\end{aligned}
$$


Then the area where $Y_{i}$ is predicted is exactly the fuzziness $\nabla \widetilde{Y}_{i}$. That is why the objective function in (33) is to minimize the total fuzziness.

Definition 6 (see [32]). The credibility of $\widetilde{Y}_{i}$ in representing $Y_{i}$ denoted by $\mathrm{Cr}_{i}$ is defined as

$$
C r_{i}=\frac{\mu_{\tilde{Y}_{i}}\left(Y_{i}\right)}{\nabla \widetilde{Y}_{i}} .
$$

and the system credibility in model (16) and (18)-(35), denoted by $\mathrm{Cr}$ is calculated as follows:

$$
\begin{aligned}
C r & =\sum_{i=1}^{N} C r_{i} \\
& =\sum_{i=1}^{N} \frac{\mu_{\widetilde{Y}_{i}}\left(Y_{i}\right)}{\nabla \widetilde{Y}_{i}} .
\end{aligned}
$$

The higher the $C r_{i}$ (resp., $C r$ ) is the better the performance of $\widetilde{Y}_{i}$ (resp., FLR) will be.

Definition 7 (from [32]).

(1) Define $S_{i}^{h^{l}}$ by

$$
S_{i}^{h^{l}}=\left\{\begin{array}{cc}
\frac{\widehat{Y}_{i}^{c}-Y_{i}}{\left(Y_{i}^{L}\right)^{h^{l *}}}=\frac{\sum_{j=0}^{m} \widehat{\alpha}_{j}^{c} x_{i j}-Y_{i}}{\left(\beta_{0}^{L}\right)^{h^{l *}}+\sum_{\substack{j=1 \\
x_{i j} \geqslant 0}}^{m}\left(\beta_{j}^{L}\right)^{h^{l *}} x_{i j}+\sum_{j=1}^{m}\left(\beta_{j}^{R}\right)^{h^{l *}} x_{i j}} & \text { if } Y_{i} \leqslant \widehat{Y}_{i}^{c} \\
\frac{Y_{i}-\widehat{Y}_{i}^{c}}{\left(Y_{i}^{R}\right)^{h^{l *}}}=\frac{\sum_{j=0}^{m} \widehat{\alpha}_{j}^{c} x_{i j}}{\left(\beta_{0}^{R}\right)^{h^{h^{*}}}+\sum_{\substack{j=1 \\
x_{i j} \geqslant 0}}^{m}\left(\beta_{j}^{R}\right)^{h^{h_{*}}} x_{i j}+\sum_{\substack{j=1 \\
x_{i j}<0}}^{m}\left(\beta_{j}^{L}\right)^{h^{l *}} x_{i j}} & \text { if } Y_{i}>\widehat{Y}_{i}^{c} \\
i=1, \ldots, N ; l=1,2 &
\end{array}\right.
$$

(2) Define $C r_{i}^{h^{l}}$ by

$$
\begin{aligned}
C r_{i}^{h^{l}}= & \frac{\left(\mu_{\widetilde{Y}_{i}}\left(Y_{i}\right)\right)^{h^{l *}}}{\nabla \widetilde{Y}_{i}^{h^{l *}}} \\
= & \frac{2\left(1-S_{i}^{h^{l *}}\right)}{\sum_{j=0}^{m}\left(\left(\beta_{j}^{L}\right)^{h^{l *}}+\left(\beta_{j}^{R}\right)^{h^{l *}}\right)\left|x_{i j}\right|}, \\
& \quad i=1, \ldots, N ; l=1,2
\end{aligned}
$$

(3) Define $p_{i}^{h^{1}}$ by

$$
p_{i}^{h^{l}}=\frac{S_{i}^{h^{l}}}{\nabla \widetilde{Y}_{i}^{h^{l *}}}, \quad i=1, \ldots, N ; l=1,2 .
$$

(4) Define $p^{h^{l}}$ by

$$
p^{h^{l}}=\sum_{i=1}^{N} p_{i}^{h^{l}}, \quad l=1,2 .
$$

\subsubsection{Optimizing the $h$ Value}

Theorem 8. Consider $h^{1}=0$ and $h^{2}=h$. Then, the optimal value of $h$ is given by

$$
h^{*}= \begin{cases}\frac{1}{2}\left(1-\frac{C r^{0}}{p^{0}}\right) & \text { if } 0 \leqslant \frac{C r^{0}}{p^{0}} \leqslant 1 \\ 0 & \text { if } \frac{C r^{0}}{p^{0}}>1\end{cases}
$$

Proof. (see [32]).

Remark 9. Reference [32] has shown that $h^{*} \leqslant 0.5$

\section{Estimation of Loss Reserve with Log-Poisson Regression}

"In this section, we are interested in the estimation of loss reserve using a GLM (log-Poisson). We consider a non-life insurance company which sells policies in a period of time (year). This year is referred as underwriting year. The claims regarding an underwriting year will not necessarily all be paid within this year. Due to legal issues, general consideration of the claim, the delay from the claim's occurrence time to the reporting time, ..., some claims are reported and paid in the following years."

At some point in time there will however be no more payments regarding underwriting year one; the year one is said to have run off. We use then the historical data of claims presented as a run-off triangle (Table 1), where $Y_{i j}$ is the total loss regarding the underwriting period $i$ which have been paid with $j$ periods delay. The loss amounts $Y_{i j}$ with $i+j=k$ have been paid in calendar year $k \in \mathbb{N}$. At period $s \in \mathbb{R}$, we have observed the payments

$$
Y_{i j}, \quad(i, j) \in \mathscr{T}_{s}
$$

where

$$
\mathscr{T}_{s}=\left\{(i, j) \in \mathbb{N}^{*} \times \mathbb{N}: 1 \leqslant i+j \leqslant s\right\}
$$

Table 1 is usually called run-off triangle, for example, in $s_{0} \in \mathbb{N}$ periods because $\forall j>s_{0}, Y_{i j}=0$. And then the reserve $R_{i}$ for the underwriting period $i$ is defined as the predictor of the 
TABLE 1: Triangle of observed incremental payments.

\begin{tabular}{|c|c|c|c|c|c|c|c|c|c|c|}
\hline & \multicolumn{10}{|c|}{ Development Year } \\
\hline & & 0 & 1 & $\ldots$ & $l$ & $\ldots$ & $k-i$ & $\ldots$ & $k-1$ & $k$ \\
\hline \multirow{8}{*}{ Accident Year } & 0 & $Y_{0,0}$ & $Y_{0,1}$ & $\ldots$ & $Y_{0, l}$ & $\ldots$ & $Y_{0, k-i}$ & $\ldots$ & $Y_{0, k-1}$ & $Y_{0, k}$ \\
\hline & 1 & $Y_{1,0}$ & $Y_{1,1}$ & $\ldots$ & $Y_{1, l}$ & $\ldots$ & $Y_{1, k-i}$ & $\ldots$ & $Y_{1, k-1}$ & \\
\hline & $\vdots$ & $\vdots$ & $\vdots$ & $\ldots$ & $\vdots$ & $\ldots$ & $\vdots$ & & & \\
\hline & $i$ & $Y_{i, 0}$ & $Y_{i, 1}$ & $\ldots$ & $Y_{i, l}$ & $\ldots$ & $Y_{i, k-i}$ & & & \\
\hline & $\vdots$ & $\vdots$ & $\vdots$ & $\ldots$ & $\vdots$ & & & & & \\
\hline & $k-l$ & $Y_{k-l, 0}$ & $Y_{k-l, 1}$ & $\ldots$ & $Y_{i, k-i}$ & & & & & \\
\hline & $\vdots$ & $:$ & $\vdots$ & & & & & & & \\
\hline & $\begin{array}{c}k-1 \\
k\end{array}$ & $\begin{array}{c}Y_{k-1,0} \\
Y_{k, 0}\end{array}$ & $Y_{k-1,1}$ & & & & & & & \\
\hline
\end{tabular}

TABLE 2: Numerical example from [25].

\begin{tabular}{ccccccc}
\hline \multicolumn{1}{c}{} & & \multicolumn{4}{c}{ Development Year } \\
\\
\hline \multirow{4}{*}{ Accident Year } & & 0 & 1 & 2 & 3 & 3130 \\
& 2000 & 1120 & 2090 & 2610 & 2920 & \\
& 2001 & 1030 & 1920 & 2370 & 2710 & \\
& 2002 & 1090 & 2140 & 2610 & & \\
& 2003 & 1300 & 2650 & & & \\
\hline
\end{tabular}

not yet observed amount $Y_{i, k-1}+\ldots, Y_{i, s_{0}}$. The total amount reserve $R$ is defined as the prediction of

$$
\sum_{i, j} Y_{i, j}, \quad(i, j) \in Q_{k}
$$

where

$$
\begin{array}{r}
\mathbb{Q}_{k} \\
\quad=\{(i, j) \in\{1, \ldots, k\} \times\{0,1, \ldots, k\}: i+j \geqslant k+1\} .
\end{array}
$$

Here we assume that $Y_{i j}$ follow a Poisson distribution with the underwriting period $i$ which is reported with $j$ period delay.

Assume that $Y_{i j},(i, j) \in \mathscr{T}_{k} \cup \mathscr{Q}_{k}$, are mutually independent [33] and

$$
\begin{aligned}
Y_{i j} & \sim \mathscr{P}\left(e^{v_{i j}}\right), \quad \text { with } \mathbb{V}\left(Y_{i j}\right)=\psi \mathbb{E}\left(Y_{i j}\right) \\
\text { i.e } \ln \mathbb{E}\left(Y_{i j}\right) & =v_{i j}
\end{aligned}
$$

where

$$
\begin{aligned}
& v_{i j}=\tau+\alpha_{i}+\beta_{j}, \quad(i, j) \in \mathscr{T}_{k} \cup \mathscr{Q}_{k}, \\
& \alpha_{1}=\beta_{0}=0,
\end{aligned}
$$

$\psi$ is the dispersion parameter, $\tau$ means that we assume the portfolio to grow, or shrink, by a fixed percentage each year, $\alpha_{i}$ means that the proportion settled decreases by a fixed fraction with each origin year, and $\beta_{j}$ means that the proportion settled decreases by a fixed fraction with each development year.
The parameter vector is given by

$$
\boldsymbol{\theta}=\left(\alpha_{1}, \ldots, \alpha_{k}, \beta_{0}, \ldots, \beta_{k-1}, \tau\right) \in \mathbb{R}^{2 k-1}
$$

The estimator of $Y_{i j}$ can be given using the following relationship:

$$
\widehat{Y}_{i j}=e^{\widehat{\tau}+\widehat{\alpha}_{i}+\widehat{\beta}_{j}}
$$

where $\widehat{\tau}, \widehat{\alpha}_{i}, \widehat{\beta}_{j}$ are the Maximum Likelihood Estimators (MLE) of $\tau, \alpha_{i}, \beta_{j}$, respectively, and could be derived from a recursive algorithm (see [7]).

Let us consider the numerical run-off triangles used in [10, 25] (see Tables 2 and 3).

As an example of reading a run-off triangle (see Table 2), we can say that 1030 is the indemnity amount of an accident occurring in 2001 and paid during the same year. 1920 is the indemnity amount of a claim occurring in 2001 and paid in 2002. The same interpretation can be done for Table 3 .

Let us plot the data (Table 2) to start the reserving analysis. The interpretation given for Table 2 could be done for Table 3.

Figure 2 presented the incremental and cumulative claims development by origin year. The run-off triangle appears to be fairly well behaved. The past years, 2003 and 2004, appear to be slightly higher than years 2000 to 2002, and the values in 2001 are lower in comparison to the later years and 2000, for example, the book changed over the years. The last payment of 2,650 for the 2003 origin year stands out a bit as well.

We performed the classical log-Poisson regression on the dataset in Tables 2 and 3, respectively, using the software R. We got the following Maximum Likelihood Estimations 
TABLE 3: Numerical example from [10].

\begin{tabular}{|c|c|c|c|c|c|c|c|c|}
\hline \multirow[b]{2}{*}{$i / j$} & & \multicolumn{7}{|c|}{ Development Year } \\
\hline & & 1 & 2 & 3 & 4 & 5 & 6 & 7 \\
\hline \multirow{7}{*}{ Accident Year } & 2007 & 3511 & 3215 & 2266 & 1712 & 1059 & 587 & 340 \\
\hline & 2008 & 4001 & 3702 & 2278 & 1180 & 956 & 629 & \\
\hline & 2009 & 4355 & 3932 & 1946 & 1522 & 1238 & & \\
\hline & 2010 & 4295 & 3455 & 2023 & 1320 & & & \\
\hline & 2011 & 4150 & 3747 & 2320 & & & & \\
\hline & 2012 & 5102 & 4548 & & & & & \\
\hline & 2013 & 6283 & & & & & & \\
\hline
\end{tabular}
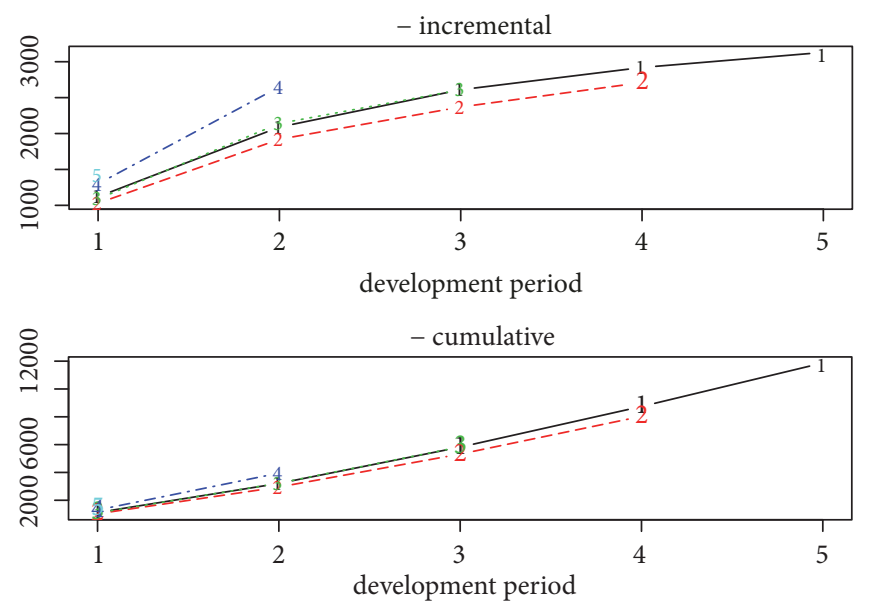

Figure 2: Description of the data (Table 2).

(MLE) of the parameters of the model and their confidence interval (CI) in Table 4.

From Table 4 (first column) and with a threshold of $5 \%$, we conclude that except $\widehat{\alpha}_{3}$, the others coefficients are statistically significant. As an example of interpretation of the results, the estimation of the payment of $2^{\text {nd }}$ origin year could be $e^{6.996-0.084+0.661}=1945.9002$. From the second column of Table 4, we conclude that all the coefficients of the model are statistically significant at a threshold of $5 \%$.

Now let us test if the model performed in each data is adapted to a statistical perspective through a dispersion test (see Table 5).

From the first column of Table 5 and with a threshold of $5 \%$, we do not reject the null hypothesis since $p-$ value $>$ $5 \%$. Therefore we do not need to perform a quasi-Poisson regression. However in the second column of Table 5 , we reject the null hypothesis with a threshold of $5 \%$, i.e., a quasiPoisson model, with the variance proportional to the mean, should be more reasonable for the data from Table 3 .

Thus the predicted incremental payments from the runoff triangle of Table 2 are displayed in Table 6.

The value of the MSEP got from the dataset 1 (Table 2) by fitting the classical log-Poisson regression model is $\mathbf{M S E P}_{\mathbf{1}}=$ 1264599. As reminder from our previous paper [1], the value of the MSEP by fitting the hybrid log-Poisson regression model (with an analytical estimation of parameters) on dataset 1 (Table 2) is $\operatorname{MSEP}_{\mathbf{F}}^{\mathbf{1}^{\prime}}=$ 699.6663. Furthermore the value of $\mathbf{R}_{1}^{2}$ from the classical log-Poisson regression model is $\mathbf{R}_{\mathbf{1}}^{\mathbf{2}}=\mathbf{9 6 . 2 1} \%$. We shall compare the values of MSEP $_{1}$ and MSEP$_{\mathbf{F}}^{\mathbf{1}^{\prime}}$ with the one we will get from the hybrid model where the fuzzy coefficients are estimated through a quadratic optimization program with optimized $h$-value $\left(\right.$ MSEP$_{\mathrm{F}}^{1}$ ). From Table 6, we compute the outstanding reserve, i.e., $\widehat{\mathbf{R}}_{1}=\sum_{i+j>5} \widehat{\mathbf{Y}}_{i j}=33634.89$.

To predict the incremental payments from Table 3, we need first to perform a quasi-Poisson regression as it has been point out in the overdispersion test (Table 5). For that data, the predicted incremental payments computed from the quasi-Poisson model are displayed in Table 7.

From Table 7, the MSEP from the classical log-Poisson regression can be computed, i.e., $\mathbf{M S E P}_{2}=\mathbf{2 5 7 4 6 . 9 5}$. Similarly, from our previous results [1], the hybrid model with analytical estimation of parameters fitted on dataset 2 (Table 3) gives as value of MSEP MSEP$_{\mathbf{F}}^{\mathbf{2}^{\prime}}=3209.07$. The goodness of fit index from the classical log-Poisson regression fitted on dataset 2 (Table 3 ) is $\mathbf{R}_{2}^{2}=\mathbf{9 7 . 7 7 \%}$, i.e., $\mathbf{9 7 . 7 7 \%}$ of the variability is explained by the model. The values of the MSEP ( $\mathbf{M S E P}_{2}, \mathbf{M S E P}_{\mathbf{F}}^{\mathbf{2}^{\prime}}$ ) will be compared with the one from the hybrid log-Poisson model where the fuzzy coefficients are estimated through a quadratic optimization program with optimized $h$-value, i.e., $\mathbf{M S E P}_{\mathbf{F}}^{2}$. From Table 7, the outstanding reserves are $\widehat{\mathbf{R}}_{2}=\sum_{\mathbf{i}+\mathbf{j}>7} \widehat{\mathbf{Y}}_{\mathbf{i j}}=$ 104327.8. 
TABLE 4: Estimated parameters of model (equation (53)).

\begin{tabular}{|c|c|c|c|c|c|c|c|}
\hline \multicolumn{4}{|c|}{ Data of Table 2} & \multicolumn{4}{|c|}{ Data of Table 3} \\
\hline$\left(\widehat{\alpha}_{i}\right)_{2 \leqslant i \leqslant 5}$ & $\left(\widehat{\beta}_{j}\right)_{2 \leqslant j \leqslant 5}$ & $p-\operatorname{value}\left(\widehat{\alpha}_{i}\right)$ & $p-\operatorname{value}\left(\widehat{\beta}_{j}\right)$ & $\left(\widehat{\alpha}_{i}\right)_{2 \leqslant i \leqslant 7}$ & $\left(\widehat{\beta}_{j}\right)_{2 \leqslant j \leqslant 7}$ & $p-\operatorname{value}\left(\widehat{\alpha}_{i}\right)$ & $p-\operatorname{value}\left(\widehat{\beta}_{j}\right)$ \\
\hline-0.084 & 0.661 & $4.23 \times e^{-08}$ & $<2 \times e^{-16}$ & 0.031 & -0.117 & 0.012 & $<2 \times e^{-16}$ \\
\hline 0.005 & 0.865 & 0.741 & $<2 \times e^{-16}$ & 0.100 & -0.628 & $2.02 \times e^{-15}$ & $<2 e-16$ \\
\hline 0.207 & 0.987 & $<2 \times e^{-16}$ & $<2 \times e^{-16}$ & 0.035 & -1.032 & 0.008 & $2 \times e^{-16}$ \\
\hline \multirow[t]{3}{*}{0.262} & 1.052 & $4.72 \times e^{-16}$ & $<2 \times e^{-16}$ & 0.089 & -1.313 & $5.36 e-11$ & $<2 \times e^{-16}$ \\
\hline & & & & 0.281 & -1.863 & $<2 \times e^{-16}$ & $<2 \times e^{-16}$ \\
\hline & & & & 0.488 & -2.428 & $<2 \times e^{-16}$ & $<2 \times e^{-16}$ \\
\hline \multicolumn{2}{|c|}{$\widehat{\tau}=6.996$} & \multicolumn{2}{|c|}{$p-\operatorname{value}(\widehat{\tau})=<2 \times e^{-16}$} & \multicolumn{2}{|c|}{$\widehat{\tau}=8.257$} & \multicolumn{2}{|c|}{$p-\operatorname{value}(\widehat{\tau})=<2 \times e^{-16}$} \\
\hline \multicolumn{2}{|c|}{ MSEP $=520.568$} & \multicolumn{2}{|c|}{ Total Reserve $=33634.89$} & \multicolumn{2}{|c|}{ MSEP $=25746.95$} & \multicolumn{2}{|c|}{ Total Reserve $=104327.8$} \\
\hline
\end{tabular}

TABLE 5: Overdispersion test.

\begin{tabular}{lc}
\hline Data from Table 2 & Data from Table 3 \\
\hline$H_{0}: \psi=1$ & $H_{0}: \psi=1$ \\
$H_{1}: \psi>1$ & $H_{1}: \psi>1$ \\
$Z=-6.3414$ & $Z=2.9661$ \\
$p-$ value $=1$ & $p-$ value $=0.001508$ \\
\hline
\end{tabular}

Remark 10. We notice that in both cases (results on dataset 1 (Table 2) and dataset 2 (Table 3), respectively), the MSEP from the hybrid model with analytical estimation of parameters is greater than the one we are getting from the classical log-Poisson regression model. However these values $\left(\operatorname{MSEP}_{\mathbf{F}}^{\mathbf{1}^{\prime}}, \mathbf{M S E P}_{\mathrm{F}}^{\mathbf{2}^{\prime}}\right)$ still be large.

After performing the classical log-Poisson model and the hybrid model with analytical estimation of parameters on each of the run-off triangles data (Tables 2 and 3), let us propose a new method to estimate the fuzzy parameters of the hybrid model and compare it with the classic logPoisson regression model and the hybrid one with analytical estimation of parameters through the same datasets" [1].

\section{Main Results}

5.1. A Quadratic Optimization Program to Estimate the Hybrid Log-Poisson Regression Model for Optimal Loss Reserving. In this section, we present a new way to estimate the hybrid logPoisson regression [1]. For that, let us define the framework where our method will be defined. Indeed, [7] has assumed that incremental payments $Y_{i j}$ are log-Poisson distributed, i.e.,

$$
Y_{i j} \sim \mathscr{P}\left(e^{\nu_{i j}}\right) \Longrightarrow \mathbb{E}\left(Y_{i j}\right)=e^{\nu_{i j}} \quad \forall(i, j) \in \mathscr{T}_{k} .
$$

Denote

$$
\mathbb{E}\left(Y_{i j}\right)=\lambda_{i j}, \quad \forall(i, j) \in \mathscr{T}_{k} .
$$

So the model becomes

$$
\ln \left(\lambda_{i j}\right)=\tau+\alpha_{i}+\beta_{j}, \quad \forall(i, j) \in \mathscr{T}_{k} .
$$

In our case let us consider the following assumptions:
Assumption 1. We consider a hybrid log-Poisson regression estimated from minimum distance of spreads and OLS criterion. Indeed, [30] considered a minimum fuzziness criterion, but in our model we consider the minimum distance between the left and right spread, i.e., the minimum of the sum of square of fuzziness as this can be seen as the well known minimum of the sum of square of errors in OLS.

Assumption 2. We assume that uncertainty about incremental payments in the run-off triangle is due to both fuzziness and randomness. Then the estimate $\hat{\lambda}_{i j}$ of $\lambda_{i j}$ will be obtain by the use of the expected value of FN on $\widetilde{\widehat{\lambda}}_{i j}$, where $\widetilde{\widehat{\lambda}}_{i j}$ denote the estimate of the fuzzy parameter $\widetilde{\lambda}_{i j}$.

We suppose that $\tilde{Y}_{i j}$ is a fuzzy Poisson random variable [34], i.e.,

$$
\widetilde{Y}_{i j} \sim \widetilde{\mathscr{P}}\left(\lambda_{i j}\right), \quad \forall(i, j) \in \mathscr{T}_{k}
$$

According to [34], the fuzzy expected value of $\widetilde{Y}_{i j}$ is defined by its $h$-level, i.e.,

$$
\begin{aligned}
{\left[\mathbb{E}_{F}\left(\tilde{Y}_{i j}\right)\right]_{h} } & =\left\{\sum_{x=0}^{+\infty} x e^{-\lambda_{i j}} \frac{\left(\lambda_{i j}\right)^{x}}{x !} \mid \lambda_{i j} \in\left[\tilde{Y}_{i j}\right]_{h}\right\} \\
& =\left\{\lambda_{i j} \mid \lambda_{i j} \in\left[\tilde{Y}_{i j}\right]_{h}\right\}=\tilde{\lambda}_{i j},
\end{aligned}
$$

where $\mathbb{E}_{F}(\cdot)$ is the fuzzy mean operator. So the fuzzy mean is just the fuzzification of the crisp mean.

Then suppose that in the log-Poisson model (57)-(58), $\lambda_{i j}$ are FN such that $\tilde{\lambda}_{i j}=\left(\lambda_{i j}^{L}, \lambda_{i j}^{c}, \lambda_{i j}^{R}\right)$ and $\mu_{\tilde{\lambda}_{i j}}$ the membership function of $\tilde{\lambda}_{i j}$, where

$$
\begin{aligned}
& \mu_{\tilde{\lambda}_{i j}}\left(\lambda_{i j}^{L}\right)=\mu_{\tilde{\lambda}_{i j}}\left(\lambda_{i j}^{R}\right)=0 \\
& \mu_{\tilde{\lambda}_{i j}}\left(\lambda_{i j}^{c}\right)=1 ; \quad \lambda_{i j}^{L}, \lambda_{i j}^{c}, \lambda_{i j}^{R} \in \mathbb{R}_{+}^{*}
\end{aligned}
$$


TABLE 6: Predicted incremental payments of the run-off triangle Table 2.

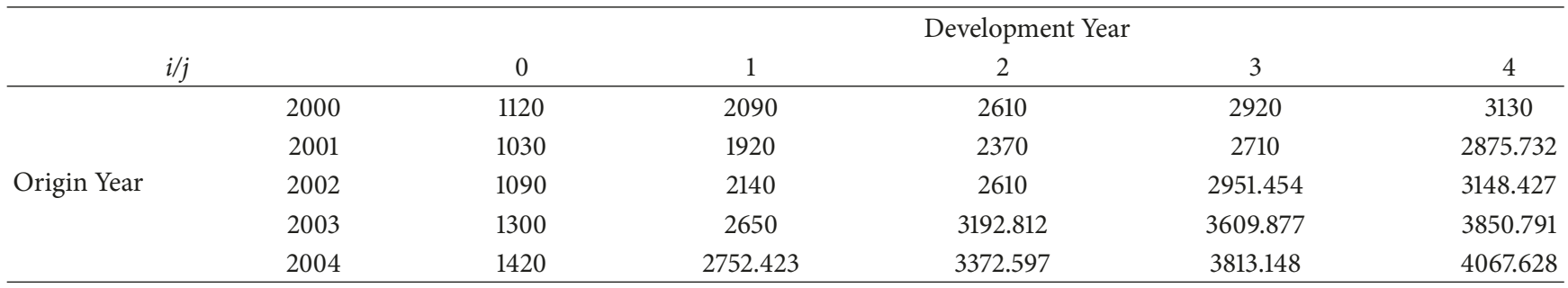

TABLE 7: Predicted incremental payments from run-off triangle Table 3.

\begin{tabular}{|c|c|c|c|c|c|c|c|c|}
\hline \multirow[b]{2}{*}{$i / j$} & & \multicolumn{7}{|c|}{ Development Year } \\
\hline & & 1 & 2 & 3 & 4 & 5 & 6 & 7 \\
\hline \multirow{7}{*}{ Accident Year } & 2007 & 3511 & 3215 & 2266 & 1712 & 1059 & 587 & 340 \\
\hline & 2008 & 4001 & 3702 & 2278 & 1180 & 956 & 629 & 350.9020 \\
\hline & 2009 & 4355 & 3932 & 1946 & 1522 & 1238 & 661.6201 & 375.9167 \\
\hline & 2010 & 4295 & 3455 & 2023 & 1320 & 1073.335 & 619.5253 & 351.9994 \\
\hline & 2011 & 4150 & 3747 & 2320 & 1502.970 & 1134 & 654.5405 & 371.8942 \\
\hline & 2012 & 5102 & 4548 & 2724.981 & 1820.420 & 1373.517 & 792.7891 & 450.4438 \\
\hline & 2013 & 6283 & 5587.059 & 3351.885 & 2239.222 & 1689.505 & 975.1765 & 554.0719 \\
\hline
\end{tabular}

Thus we define the membership function of $\tilde{\lambda}_{i j}$ as follows:

$$
\mu_{\tilde{\lambda}_{i j}}(x)= \begin{cases}1-\frac{\lambda_{i j}^{c}-x}{\lambda_{i j}^{L}} & \text { if } \lambda_{i j}^{c}-\lambda_{i j}^{L}<x \leqslant \lambda_{i j}^{c} \\ 1-\frac{x-\lambda_{i j}^{c}}{\lambda_{i j}^{R}} & \text { if } \lambda_{i j}^{c}<x \leqslant \lambda_{i j}^{c}+\lambda_{i j}^{R} \\ 0 & \text { if otherwise }\end{cases}
$$

Denote $\ln \left(\widetilde{\lambda}_{i j}\right)=\widetilde{v}_{i j}$.

Then the hybrid model built over the log-Poisson regression can have an alternative definition as follows:

$$
\widetilde{Y}_{i j} \sim \widetilde{\mathscr{P}}\left(e^{\tilde{\nu}_{i j}}\right), \quad \widetilde{v}_{i j}=\widetilde{\tau}+\widetilde{\alpha}_{i}+\widetilde{\beta}_{j}, \quad \forall(i, j) \in \mathscr{T}_{k},
$$

and $\widetilde{\alpha}_{1}=\widetilde{\beta}_{1}=0_{\mathbb{R}^{3}}$

where

$$
\widetilde{v}_{i j}=\left(v_{i j}^{L}, v_{i j}^{c}, v_{i j}^{R}\right) \in \mathbb{R}^{3}
$$

are ATFN and

$$
\begin{aligned}
& v_{i j}^{L}=\tau^{L}+\alpha_{i}^{L}+\beta_{j}^{L} \\
& v_{i j}^{c}=\tau^{c}+\alpha_{i}^{c}+\beta_{j}^{c} \\
& v_{i j}^{R}=\tau^{R}+\alpha_{i}^{R}+\beta_{j}^{R}
\end{aligned}
$$

The goal is to provide a method to estimate the fuzzy parameters $\widetilde{v}_{i j}$ of model (64) that is the purpose of the next subsection.

5.2. Modeling Methodology Setup. The steps for implementing the new model can be carried out as follows:
(1) Modeling of the centres of fuzzy parameters: we apply the classical log-Poisson Regression to the incremental payments $Y_{i j}$ of Tables 2 and 3, respectively; i.e., we assume that $\left\{Y_{i j}: i=1, \ldots, k ; j=1, \ldots, k-i+1\right\}$ are modelled by

$$
\begin{aligned}
& Y_{i j} \sim \mathscr{P}\left(e^{\nu_{i j}}\right)=\mathscr{P}\left(e^{\tau+\alpha_{i}+\beta_{j}}\right) \\
& \Longleftrightarrow v_{i j}=\ln \mathbb{E}\left(Y_{i j}\right) \\
& \Longleftrightarrow \mathbb{E}\left(Y_{i j}\right)=e^{X_{i}^{T} \boldsymbol{\theta}} \\
& \Longleftrightarrow \ln \mathbb{E}\left(Y_{i j}\right)=X_{i}^{T} \boldsymbol{\theta} \\
& \Longleftrightarrow \ln \mathbb{E}(\mathbf{Y})=\mathbf{X} \boldsymbol{\theta} \\
& \mathbf{Y}=\boldsymbol{\varepsilon} e^{\mathbf{X} \boldsymbol{\theta}}
\end{aligned}
$$

where

$\mathbf{X}_{(n \times p)}$ is the matrix of explanatory variables $x_{i j}$,

$\mathbf{Y}_{(n \times 1)}$ is the vector of observations $Y_{i j}$,

$\boldsymbol{\theta}_{(p \times 1)}$ is the vector of parameters $n=(1 / 2) k(k+1)$ and $p=2 k-1$.

$\boldsymbol{\varepsilon}_{n \times 1}$ is the vector of errors terms $\varepsilon_{i j}$ such that $\varepsilon_{i j} \sim$ $\mathscr{P}(1)$ and $\mathbb{E}\left(\varepsilon_{i j}\right)=\mathbb{V}\left(\varepsilon_{i j}\right)=1$.

(2) Estimation and optimization offuzzy parameters of the model: we consider the parameters of model (59) as FN and the model becomes as in (64). The centres of fuzzy coefficients of the model (64) are estimated in step (1).

Let us assume that these estimates are $\widehat{v}_{i j}^{c}$, where

$$
\widehat{v}_{i j}^{c}=\widehat{\tau}^{c}+\widehat{\alpha}_{i}^{c}+\widehat{\beta}_{j}^{c}
$$


To estimate the parameters $v_{i j}^{R}, v_{i j}^{L}, i \in\{2,3, \ldots, k\}$; $j \in\{2,3, \ldots, k-i\}$ in (64), we present the problem as a quadratic optimization problem. This can be written as follows:

$$
\begin{array}{ll}
\min : & z=\sum_{i=1}^{k} \sum_{j=1}^{k-i}\left(v_{i j}^{R}-v_{i j}^{L}\right)^{2} \\
\mathrm{~s} / \mathrm{t} \quad & h^{*} \cdot \widehat{v}_{i j}^{c}+\left(1-h^{*}\right) \cdot v_{i j}^{L} \leqslant \ln \left(Y_{i j}\right) \\
& h^{*} \cdot \widehat{v}_{i j}^{c}+\left(1-h^{*}\right) \cdot v_{i j}^{R} \geqslant \ln \left(Y_{i j}\right) \\
& v_{i j}^{L}, v_{i j}^{R} \geqslant 0, \quad i=1,2, \ldots, k ; j=1,2, \ldots, k-i
\end{array}
$$

where $h^{*}$ is the optimized value of $h$. Since [32] has proved that the optimal value $h^{*}$ of $h$ satisfies $h^{*} \epsilon$ $(0,0.5]$, our idea is to discretise the interval $(0,0.5]$ into certain $N_{\max }$ values. Then for each value of $h$, we estimate $v_{i j}^{R}, v_{i j}^{L} i \in\{2,3, \ldots, k\} ; j \in\{2,3, \ldots, k-i\}$. Then we calculate the outstanding reserves $\widehat{R}$ and the MSEP. We only return the values of $h, v_{i j}^{R}, v_{i j}^{L}, \widehat{R}$ for which MSEP is minimum.

(3) Estimation of the Mean Square Error Prediction (MSEP):

$\operatorname{MSEP}=\frac{1}{n} \sum_{i, j}\left(Y_{i j}-\widehat{Y}_{i j}\right)^{2}, \quad \widehat{Y}_{i j}=\mathbb{E}_{F}\left(\widehat{\widetilde{Y}}_{i j}, \pi\right)$

where

(i) $\pi$ is the decision-maker risk aversion parameter $(0 \leqslant \pi \leqslant 1)$,

(ii) $\mathbb{E}_{F}(\cdot)$ denotes the expected value of $\mathrm{FN}$,

(iii) $\widehat{\widetilde{Y}}_{i j}$ is the predicted fuzzy incremental payment.

(4) Estimation of the outstanding reserve: the fuzzy total loss reserve is computed as follows:

$$
\begin{aligned}
\widetilde{\widehat{R}} & =\sum_{i=1}^{k} \sum_{j=k-i+1}^{k} e^{\hat{\tilde{\nu}}_{i j}} \\
& =\left(\sum_{i=1}^{k} \sum_{j=k-i+1}^{k} e^{\hat{\nu}_{i j}^{L}}, \sum_{i=1}^{k} \sum_{j=k-i+1}^{k} e^{\widehat{\nu}_{i j}^{c}}, \sum_{i=1}^{k} \sum_{j=k-i+1}^{k} e^{\hat{\nu}_{i j}^{R}}\right) \\
& =\left(\widehat{R}^{L}, \widehat{R}^{c}, \widehat{R}^{R}\right)
\end{aligned}
$$

In this article, we use the concept of expected value of FN [35] to move from the fuzzy value of total loss reserve $\widetilde{\widehat{R}}$ to the crisp value of total loss reserve $\widehat{R}$.

The $h$-level of fuzzy total loss reserve is defined as follows:

$$
\widetilde{\widehat{R}}(h)=\left[h \cdot \widehat{R}^{c}+(1-h) \cdot \widehat{R}^{L} ; h \cdot \widehat{R}^{c}+(1-h) \cdot \widehat{R}^{R}\right]
$$

Then the expected value of FN $\widetilde{\widehat{R}}$ is defined as follows:

$$
\begin{aligned}
\mathbb{E}_{F}(\widetilde{\widehat{R}}, \pi)= & (1-\pi) \int_{0}^{1}\left(h \cdot \widehat{R}^{c}+(1-h) \cdot \widehat{R}^{L}\right) d h \\
& +\pi \int_{0}^{1}\left(h \cdot \widehat{R}^{c}+(1-h) \cdot \widehat{R}^{R}\right) d h
\end{aligned}
$$

From (73), we have

$$
\begin{aligned}
\mathbb{E}_{F}(\widetilde{\widehat{R}}, \pi) \\
=(1-\pi) \int_{0}^{1}\left(h \cdot \widehat{R}^{c}+\widehat{R}^{L}-h \cdot \widehat{R}^{L}\right) d h \\
\quad+\pi \int_{0}^{1}\left(h \cdot \widehat{R}^{c}+\widehat{R}^{R}-h \cdot \widehat{R}^{R}\right) d h \\
=(1-\pi) \int_{0}^{1} h\left(\widehat{R}^{c}-\widehat{R}^{L}\right) d h+(1-\pi) \int_{0}^{1} \widehat{R}^{L} d h \\
\quad+\pi \int_{0}^{1} h\left(\widehat{R}^{c}-\widehat{R}^{R}\right) d h+\pi \int_{0}^{1} \widehat{R}^{R} d h \\
=\frac{(1-\pi)\left(\widehat{R}^{c}-\widehat{R}^{L}\right)}{2}+(1-\pi) \widehat{R}^{L}+\frac{\pi\left(\widehat{R}^{c}-\widehat{R}^{R}\right)}{2} \\
\quad+\pi \widehat{R}^{R} \\
\quad(1-\pi)\left(\widehat{R}^{c}+\widehat{R}^{L}\right) \\
2
\end{aligned}
$$

where $\pi$ is the decision-maker risk aversion parameter $(0 \leqslant \pi \leqslant 1)$. In this paper, we have chosen the maximum risk aversion parameter, i.e., $\pi=1$.

5.3. Implementation of the Method on Data. We apply our model (64) where the parameters are estimated through a quadratic optimization program on each of the run-off triangles from [25] (see Table 2) and from [10] (see Table 3). We first fit the log-Poisson regression model on these data to estimate the centres $\left(\widehat{\nu}_{i j}^{c}\right)$ of the fuzzy parameters $\widetilde{v}_{i j}$ using $R$ software. The results are presented in Table 4.

Then we use the quadratic optimization program in (69) to estimate the left and right spreads of the fuzzy parameters of model (64). For that, we discretise the interval $(0,0.5]$ into certain $N_{\max }$ observations (for, e.g., $N_{\max }=100$ ). For each value $h_{t}$ of this sequence taken by $h$, we calculate the left and right spreads of fuzzy parameters of model (64), $\left(\widehat{v}_{i j}^{L}\right)^{h_{t}}$ and $\left(\widehat{v}_{i j}^{R}\right)^{h_{t}}$, the value of the outstanding reserves and the MSEP. At the end of the algorithm (in R), we return the optimal value of $h, h^{*}$ (for which MSEP is minimum), the outstanding reserves, and the MSEP. All this process can be presented as a graphic (see Figure 3 for the data from Table 2 and Figure 4 for the data from Table 3).

In Figure 3, we are interested in the minimum of MSEP and the corresponding values of $h$ and the outstanding reserves. From this graphic, we can see that the MSEP have 


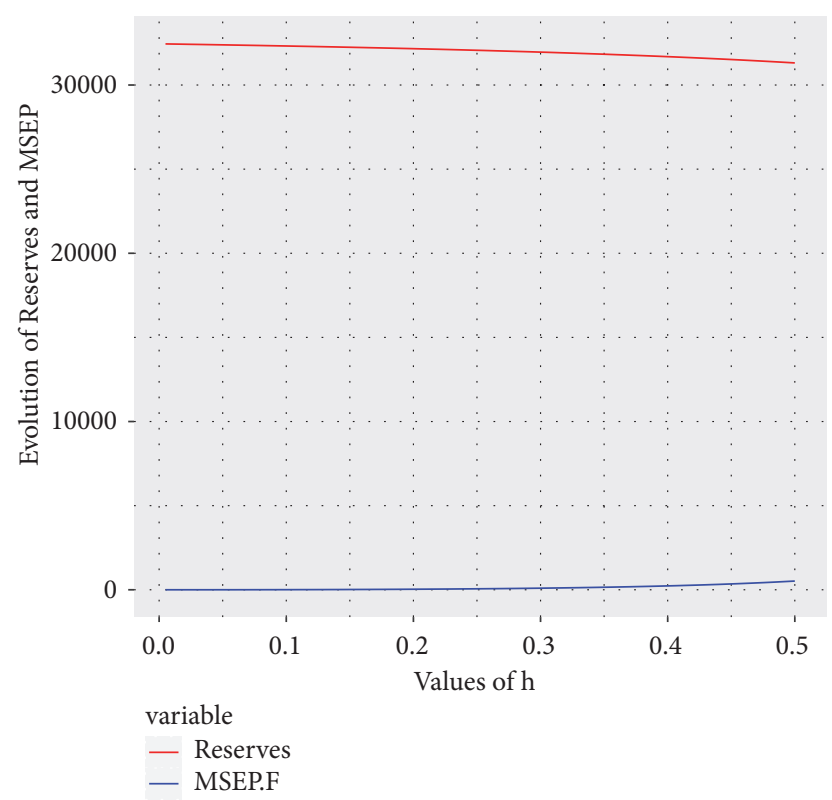

FIGURE 3: Evolution of reserves and MSEP with respect to $h$-values (data of Table 2).

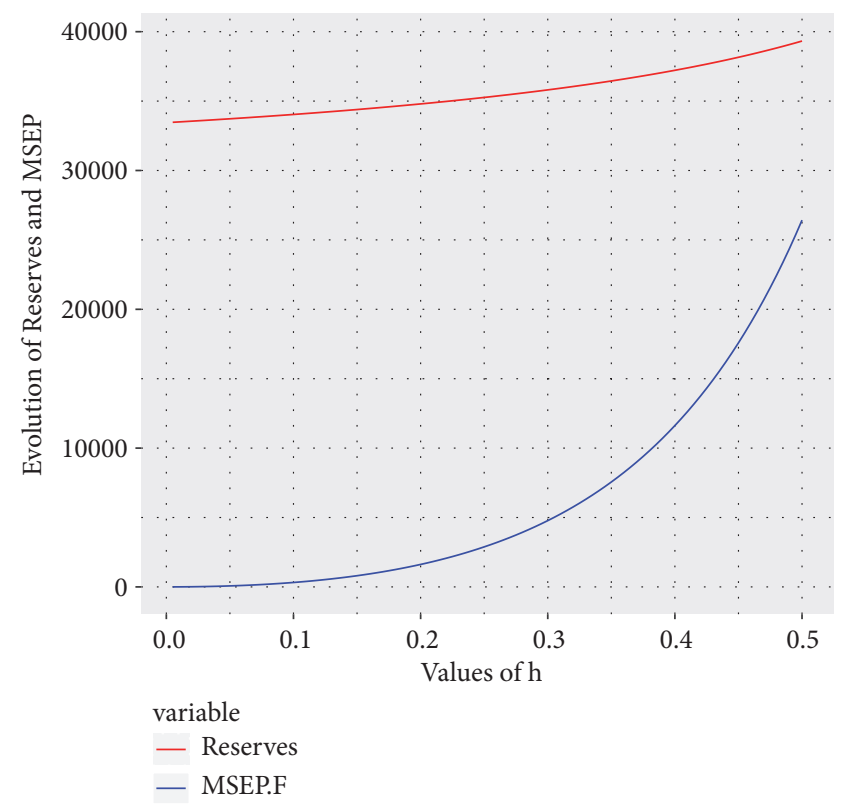

FIgURE 4: Evolution of reserves and MSEP with respect to $h$-values (data of Table 3).

two minima. Fortunately both of these minima correspond to the same reserves; i.e., we have two optimal values of $h$ but any of them will give us the same conclusions.

We can easily get these values from $R$ functions; i.e., the optimal value of $h$ is $\boldsymbol{h}_{1}^{*}=0.005$ and the corresponding mean square error prediction is $\widehat{\mathbf{M S E P}}_{\mathbf{F}}^{\mathbf{1}}=0.01313098$. The optimal outstanding reserves are $\widehat{\mathbf{R}}_{1 \mathrm{~F}}=32441.87$. In [1] and using the data displayed in Table 2, the value of the MSEP is $699.66>0.01313098$. Although the two values are lower than the one we are getting from the classical log-Poisson model $\left(\right.$ MSEP $\left._{1}=1264599\right)$, we notice that the MSEP got from the hybrid log-Poisson model estimated through the quadratic optimization program with optimized $h$-value is too low compared to the one we got in [1]. That means the fact that we have taken into account the optimized $h$-value has a positive impact on the results, namely, the power prediction of the model.

Similarly in Figure 4, we can see the evolution of mean square error prediction and reserves with respect to the values of $h$. From the figure the optimal value of $h$ is got from the first values. Using $\mathrm{R}$ functions, we get the optimal value of $h$, $\boldsymbol{h}_{2}^{*}=0.005$. The corresponding mean square error prediction is $\widehat{\mathbf{M S E P}}_{\mathbf{F}}^{2}=0.6517489$ and the optimal outstanding reserves are $\widehat{\mathbf{R}}_{2 \mathbf{F}}=33472.61$. In [1] and using the data displayed in Table 3, the value of the MSEP is $\operatorname{MSEP}_{\mathbf{F}}^{2^{\prime}}=3209.07>$ $\widehat{\operatorname{MSEP}}_{\mathrm{F}}^{2}=699.66>\widehat{\operatorname{MSEP}}_{\mathrm{F}}^{2}=0.6517489$. We conclude that the MSEP got from the hybrid log-Poisson model estimated through the quadratic optimization program with optimized $h$-value is too low compare to the one we got in [1]. Hence with the hybrid log-Poisson model estimated through the quadratic optimization program with optimized $h$-value, we are approaching the fair value of the outstanding reserve better than the classical log-Poisson regression model and the hybrid log-Poisson regression model with analytical expression of fuzzy parameters.

Remark 11 .

(1) The fact that $\widehat{\mathbf{R}}_{\mathbf{1 F}}=32441.87<33634.89=\widehat{\mathbf{R}}_{\mathbf{1}}$ and $\widehat{\mathbf{R}}_{\mathbf{2 F}}=33472.61<104327.8=\widehat{\mathbf{R}}_{\mathbf{2}}$ allows us to conclude that the classical log-Poisson model overestimates the outstanding reserves.

(2) To find the optimal value of $h$, we had to estimate the outstanding reserve and the MSEP for each value of $h$ which makes the computational part heavy.

(3) The optimization program written under the $R$ package "minqa " needs initial values of the parameters to run and converge. Therefore, we have to choose carefully these initial values; otherwise the algorithm could not find a minimum.

(4) From the outputs of numerical tests, we notice that both the optimal values $h^{*}$ are found from the earlier values of $h$. This could suggest thinking about a shorter interval where the optimal value lies and providing a mathematical proof.

\section{Conclusion}

This paper has considered the relevance of the $h$-value in hybrid models for loss reserving purpose, mainly when we are in presence of vague information like in general insurance [20]. In this article, we have considered an alternative way to estimate the fuzzy parameters of the hybrid log-Poisson regression model. To estimate these fuzzy parameters, we considered the minimum of the total square of fuzziness criterion rather than the minimum of the total fuzziness 
criterion as in [29]. This is because from Figure 1, we can see the criterion as the minimization of the distance between the left spread and the right spread. The optimized $h$-value [32] has been taken into account in the quadratic optimization problem and its relevance has been shown through a numerical test on two different datasets. It has been shown that the hybrid model estimated through the quadratic optimization program with optimized $h$-value produces best results of reserves than the classical log-Poisson regression and the hybrid model where the fuzzy parameters have an analytical form [1] according to the MSEP. Hence the outstanding reserves have been optimized

The hard computation aspect of that model constitutes a weakness compared to the simplest computation of a classical log-Poisson regression.

In order to reinforce the robustness of our model, the next work could move towards the construction of a goodness of fit index from the possibilistic approach as it has been done in [1] for the fuzzy least square approach. From the outputs of numerical examples, we notice that both the optimized $h$-values $\left(h^{*}\right)$ are found from the earlier values of $h$. This could suggest thinking about a shorter interval where the optimal $h$-value is located and providing a mathematical proof.

\section{Data Availability}

The run-off triangle data used to support the findings of this study are included within the article.

\section{Disclosure}

This manuscript has been presented in $62^{\text {nd }}$ ISI World Statistics Congress 2019 (ISI WSC 2019).

\section{Conflicts of Interest}

The authors declare that there is no conflict of interest regarding the publication of this paper.

\section{Acknowledgments}

This work was supported by African Union through Pan African University/Jomo Kenyatta University of Agriculture and Technology.

\section{Supplementary Materials}

The following are the $\mathrm{R}$ codes used for the numerical part of the article. Some R packages have been used to achieve the programme. To check the results we got in our article, one needs to install and load those packages in the $\mathrm{R}$ software. Then the code should run successfully. Therefore, inside the code, when a package is loading it is needed to install that package on your R software. (Supplementary Materials)

\section{References}

[1] W. Apollinaire, M. B. M. Le Doux, and W. M. Ronald, "A fuzzy least-squares estimation of a hybrid log-poisson regression and its goodness of fit for optimal loss reserves in insurance," International Journal of Fuzzy Systems, vol. 21, no. 3, pp. 930944, 2018.

[2] R. L. Bornhuetter and R. E. Ferguson, “The actuary and IBNR," In Proceedings of the Casualty Actuarial Society, vol. 59, no. 112, pp. 181-195, 1972.

[3] G. C. Taylor, Claims Reserving in Non-Life Insurance, vol. 1, Elsevier Science Ltd, 1986.

[4] V. E. Jacob, Loss Reserving Methods, no. 1, Research Department, Nationale-Nederlanden NV, 1981.

[5] G. Taylor, G. McGuire, and A. Greenfield, "Loss reserving: past, present and future," SSRN Electronic Journal, 2003.

[6] M. V. Wüthrich and M. Merz, Stochastic Claims Reserving Methods in Insurance, vol. 435, John Wiley \& Sons, 2008.

[7] T. Mack, "A simple parametric model for rating automobile insurance or estimating IBNR claims reserves," ASTIN Bulletin, vol. 21, no. 1, pp. 93-109, 1991.

[8] P. D. England and R. J. Verrall, "Stochastic claims reserving in general insurance," British Actuarial Journal, vol. 8, no. 3, pp. 443-518, 2002.

[9] J. Van Eeghen, Loss Reserving Methods, no. 1, Research Department, Nationale-Nederlanden NV, 1981.

[10] S. Christofides, "Regression models based on log-incremental payments," Claims Reserving Manual, vol. 2, pp. D5.1-D5.53, 2014.

[11] F. W. Accomando and E. Weissner, "Report lag distributions: estimation and application to IBNR counts," in Transcripts of the 1988 Casualty Loss Reserve Seminar, pp. 1038-1133, Casualty Actuarial Society, Arlington, VA, USA, 1988.

[12] K. H. S. Adams, "Chain ladder reserving methods for liabilities with per occurrence limits," in Casualty Actuarial Society EForum, p. 112, 2013.

[13] J. P. Nielsen, T. Agbeko, M. D. M. Miranda, and R. J. Verrall, "Validating the double chain ladder stochastic claims reserving model," Variance: Advancing the Science of Risk, vol. 8, no. 2, pp. 138-160, 2014.

[14] G. Jinadasa, "Statistical methods for health actuaries IBNR estimates: An introduction," 2007.

[15] S. Björkwall, O. Hössjer, and E. Ohlsson, "Bootstrapping the separation method in claims reserving," The Journal of the International Actuarial Association, vol. 40, no. 2, pp. 845-869, 2010.

[16] K. A. Blum and J. David, "Best estimate loss reserving: an actuarial perspective," In CAS Forum Fall, vol. 1, no. 55, p. 101, 1998.

[17] M. Buchwalder, H. Bühlmann, M. Merz, and M. V. WüTHrich, "The mean square error of prediction in the chain ladder reserving method (Mack and MURphy revisited)," The Journal of the International Actuarial Association, vol. 36, no. 2, pp. 521$542,2006$.

[18] CAS Tail Factor Working Party, "The estimation of loss development tail factors: a summary report," in Casualty Actuarial Society Forum, 2013.

[19] R. Dahms, "Linear stochastic reserving methods," The Journal of the International Actuarial Association, vol. 42, no. 1, pp. 134, 2012. 
[20] E. Straub and Swiss Association of Actuaries (Zürich), Non-Life Insurance Mathematics, no. 517/s91n, Springer, Berlin, Germany, 1988.

[21] L. A. Zadeh, "Fuzzy sets," Information and Computation, vol. 8, no. 3, pp. 338-353, 1965.

[22] D. Dubois and H. Prade, "Operations on fuzzy numbers," International Journal of Systems Science, vol. 9, no. 6, pp. 613626, 1978.

[23] D. Didier and H. Prade, "Fuzzy numbers: an overview," In Readings in Fuzzy Sets for Intelligent Systems, pp. 112-148, 1993.

[24] Y.-J. Lai and C.-L. Hwang, "Fuzzy mathematical programming," in Fuzzy Mathematical Programming, pp. 74-186, Springer, Berlin, Germany, 1992.

[25] J. de Andés-Sánchez, "Claim reserving with fuzzy regression and Taylor's geometric separation method," Insurance: Mathematics \& Economics, vol. 40, no. 1, pp. 145-163, 2007.

[26] L. A. Zadeh, "The concept of a linguistic variable and its application to approximate reasoning I," Information Sciences, vol. 8, no. 3, pp. 199-249, 1975.

[27] L. A. Zadeh, "The concept of a linguistic variable and its application to approximate reasoning-Part II," Information Sciences, vol. 8, no. 4, pp. 301-357, 1975.

[28] L. A. Zadeh, "The concept of a linguistic variable and its application to approximate reasoning-part III," Information Sciences, vol. 9, no. 1, pp. 43-80, 1975.

[29] G. F. Groner, J. F. Heafner, and T. W. Robinson, "On-line computer classification of handprinted chinese characters as a translation aid," IEEE Transactions on Electronic Computers, vol. EC-16, no. 6, pp. 856-860, 1967.

[30] H. Ishibuchi and M. Nii, "Fuzzy regression using asymmetric fuzzy coefficients and fuzzified neural networks," Fuzzy Sets and Systems, vol. 119, no. 2, pp. 273-290, 2001.

[31] A. Kaufmann and M. M. Gupta, Introduction to Fuzzy Arithmetic: Theory and Applications, D. Van Nostrand Reinhold Company, New York, NY, USA, 1991.

[32] F. Chen, Y. Chen, J. Zhou, and Y. Liu, "Optimizing h value for fuzzy linear regression with asymmetric triangular fuzzy coefficients," Engineering Applications of Artificial Intelligence, vol. 47, pp. 16-24, 2016.

[33] A. E. Renshaw and R. J. Verrall, "A stochastic model underlying the chain-ladder technique," British Actuarial Journal, vol. 4, no. 4, pp. 903-923, 1998.

[34] J. J. Buckley, Fuzzy Probability and Statistics, Springer, Heidelberg, Germany, 2006.

[35] J. de Andrés-Sánchez, "Claim reserving with fuzzy regression and the two ways of ANOVA," Applied Soft Computing, vol. 12, no. 8, pp. 2435-2441, 2012. 


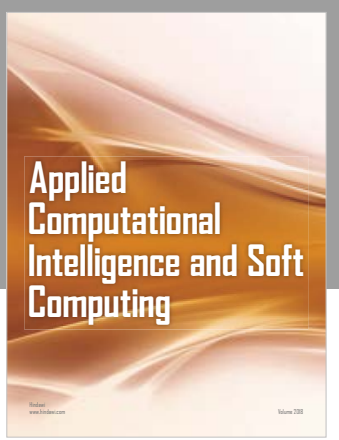

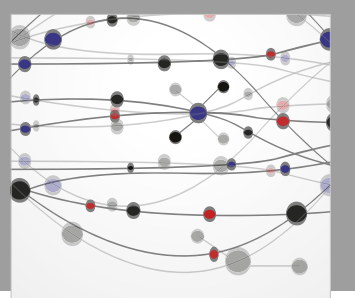

The Scientific World Journal
Submit your manuscripts at

Computing
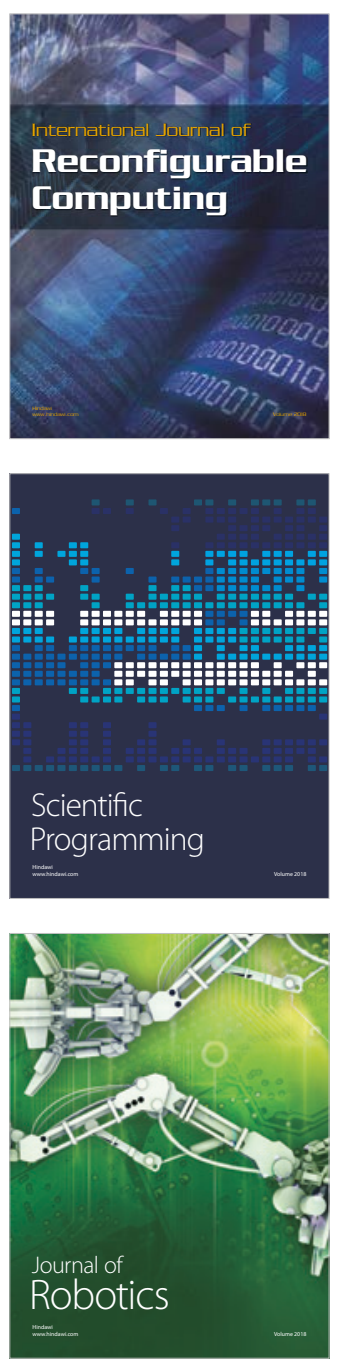

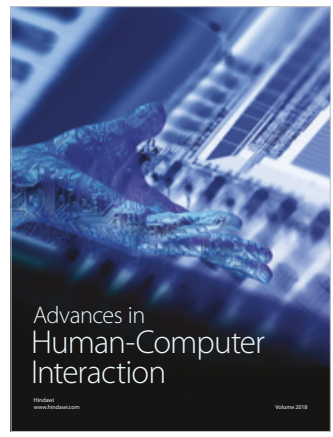

Human-Compute

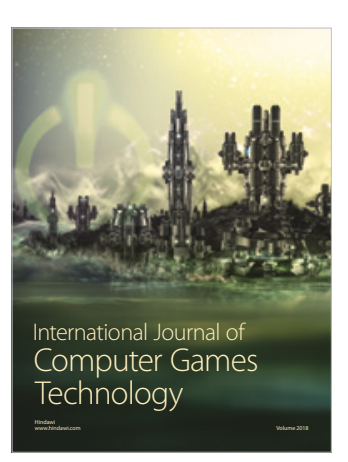

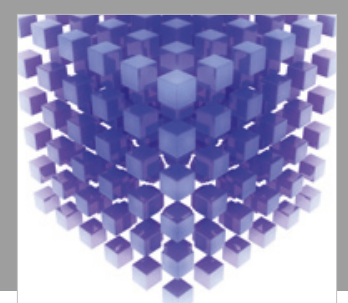

Mathematical Problems in Engineering

\section{Engincering}
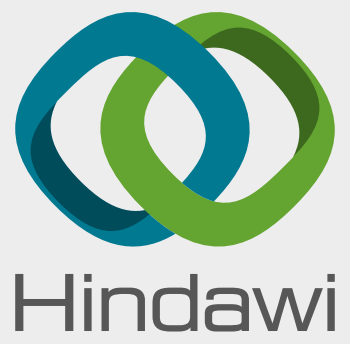

www.hindawi.com
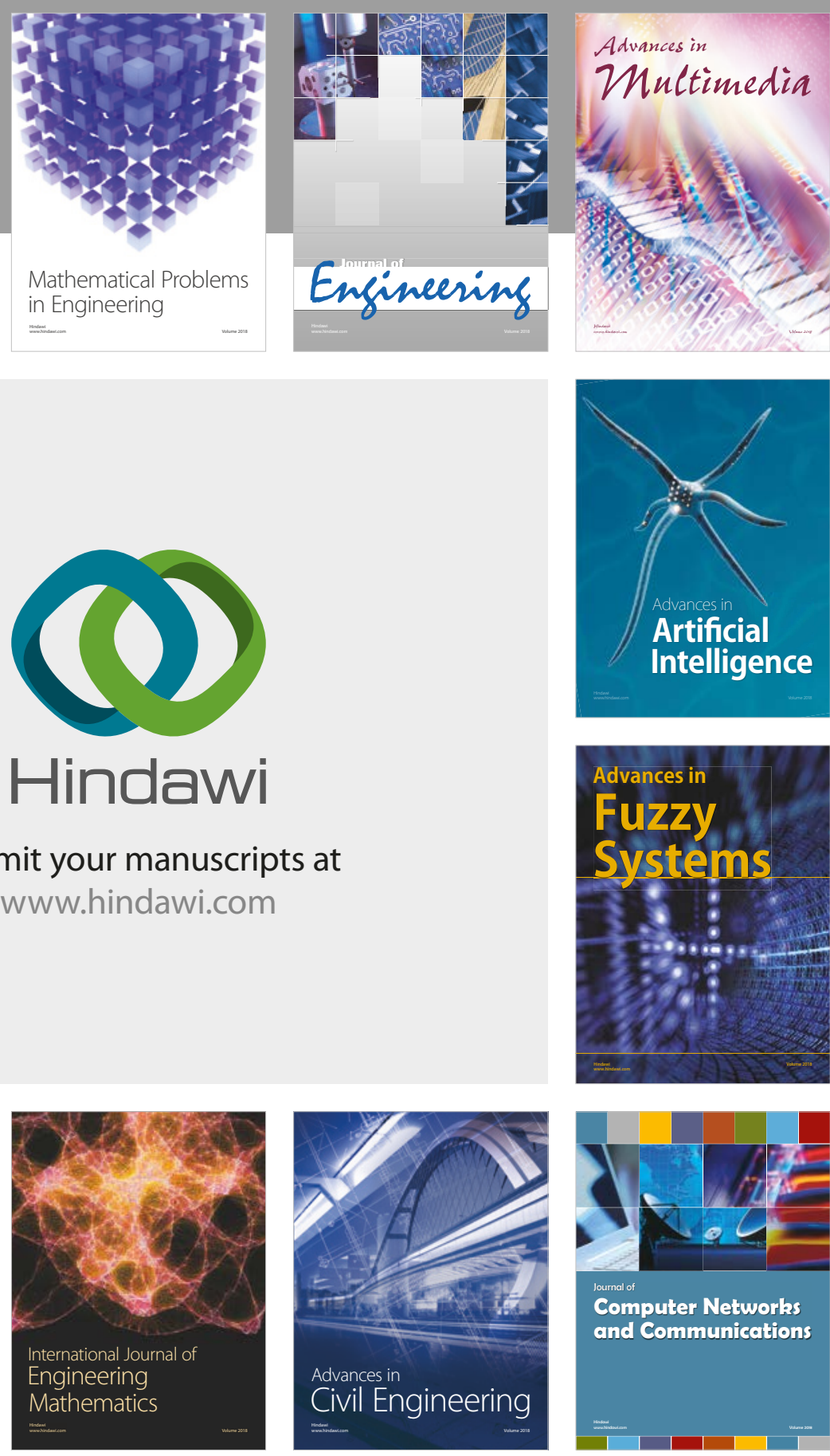

Computer Networks and Communications

Multimedia
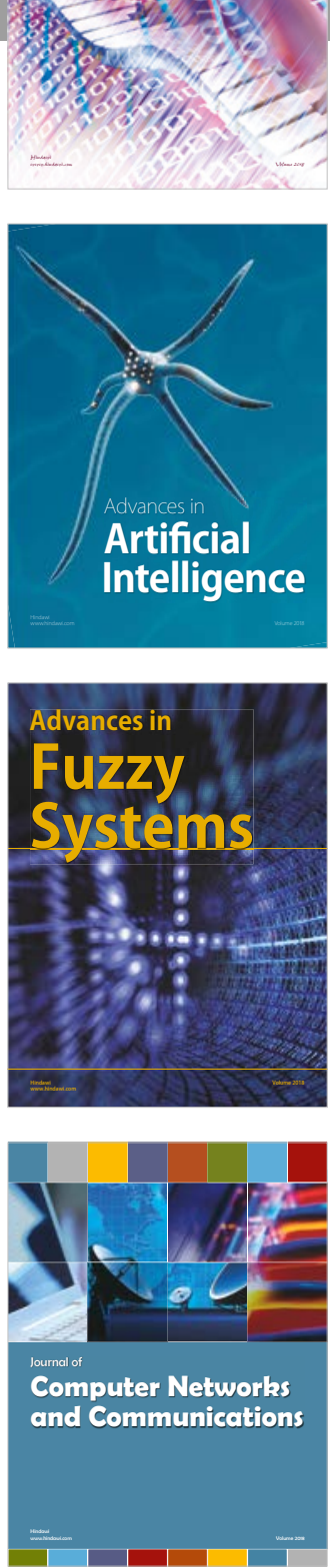

Advances in

Modelling \&

Simulation

in Engineering

interaction

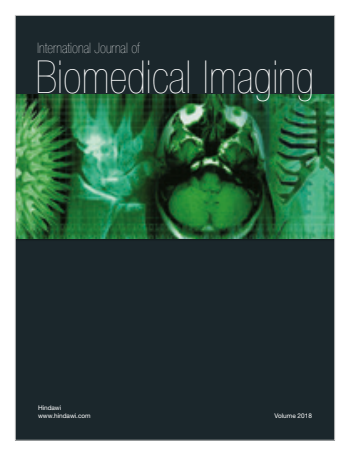

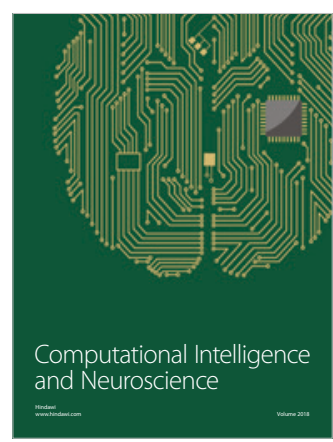

